\title{
Immunohistochemical Expression of Debiquitinating Enzyme OTUB 1 in Colorectal Carcinoma
}

\author{
NEDAL A. HEGAZY, M.D.; RIHAM M. ABU ZEID, M.D.; EMAN A. IBRAHIM, M.D. and \\ DIANA Z. SAAD, M.Sc.
}

The Department of Pathology, Faculty of Medicine, Ain Shams University, Cairo, Egypt

\begin{abstract}
Background and Objective: Colorectal Carcinoma (CRC) is the most common malignancy of the gastrointestinal tract. The overall five-year survival for colon cancer and rectal cancer are $65 \%$ and $68 \%$, respectively with liver metastasis being the leading cause of death. Despite the use of active targeted drugs for treatment of metastatic CRC in the past decade, cure rates remain low. Therefore, research on novel markers for earlier diagnosis and target therapy of CRC is of a great concern. OTUB 1 (OTU deubiquitinase, ubiquitin aldehyde binding 1) is a deubiquitinating enzyme (DUB) that belongs to the OTU (ovarian tumor) superfamily. The aim of this work is to study the immunohistochemical expression of OTUB $_{1}$ in CRC and its correlation with the available clinicopathological variables.
\end{abstract}

Material and Methods: This retrospective study was conducted on a total number of 45 cases of CRC. All cases were retrieved from the archival files of the pathology labs of Ain Shams University Hospitals (from 2012-2016). Immunohistochemistry using rabbit polyclonal anti OTUB 1 antibody was performed.

Results: OTUB 1 was overexpressed in CRC tissues, and the expression level of OTUB 1 was correlated significantly with histologic grade $(p<0.001)$, depth of invasion (pathological T) $(p=0.03)$ and location of CRC $(p=0.002)$. On the other hand, no significant correlation was found with age, gender, metastasis whether nodal or distant, TNM stage and size of tumor.

Conclusion: OTUB 1 may have a possible role in tumor differentiation as well as local tumor invasion, thus could be useful in identification of biologically aggressive tumors in colonoscopic biopsies in addition to providing a basis for targeted therapy which could play a major role in the treatment of CRC.

Key Words: OTUB 1 - Colorectal carcinoma - Immunohistochemistry.

Correspondence to: Dr. Nedal A. Hegazy, The Department of Pathology, Faculty of Medicine, Ain Shams University, Cairo, Egypt

\section{Introduction}

COLORECTAL Cancer (CRC) is the most common malignancy of the gastrointestinal tract ranking the third most commonly diagnosed malignancy in men while the second most common cancer in women and the fourth leading cause of cancer death. More than two-thirds of all cases and about $60 \%$ of all deaths occurred in countries with a high or very high human development index [1]. CRC in Egypt, as most of developing countries, has lower incidence than that of western countries with sedentary lifestyle. In Egypt, it is the sixth most common cancer constituting about $4 \%$ of total cancers in both sexes [2].

From a molecular aspect, several genes have been implicated in bladder carcinogenesis, mostly oncogenes (i.e. k-ras) and tumor suppressor genes (i.e. APC, p53, SMAD4), whose alterations lead to genetic instability and eventually to full development of histologically apparent colorectal tumors

DUBs are cysteine proteases that catalyze the removal of Ubiquitin (UB) from Ub-modified proteins and responsible for the processing of nascent translated linked Ub precursors. OTUB is a cysteine protease that hydrolyses the isopeptide bond between ubiquitin and the target molecule [4].OTUB ${ }_{1}$ has recently emerged as a unique atypical DUB that binds and inhibits several classes of E2s, thus in this way inhibits DNA repair [5] Recent studies found that DUBs are crucial in regulating a variety of cellular pathways, including cell growth and proliferation as well as apoptosis, thus are key molecular determinants of aberrant cancer proteome $[\mathbf{5 , 6 , 8 , 9 ]}$.

The overexpression of OTUB ${ }_{1}$ has been found in many human cancers such as in prostate, color- 
ectal, breast, gastric and lung cancers that are associated with poor survival, high metastatic potential, and chemotherapeutic drug resistance [10].

To date, there is a paucity of data in the relationship between OTUB ${ }_{1}$ and colorectal carcinoma. The aim of the present study is to assess the immunohistochemical expression of OTUB ${ }_{1}$ in cases of CRC and to correlate it with various clinicopathological parameters.

\section{Material and Methods}

\section{Tissue collection:}

The current study was conducted on 45 cases of colorectal carcinoma. Cases were retrieved from archives of the Pathology Department, Ain-Shams University Hospital during the period from January 2012 to December 2016. They were obtained by radical colectomy. The surgical and histopathology reports were reviewed to determine age, sex of the patients as well as the histologic type of CRC. In addition, 10 cases of non-neoplastic colonic lesions were obtained by colonoscopic biopsies included as a control.

Haematoxylin and Eosin stained slides were examined for the confirmation of the diagnosis. Examination of the tumor was done for pathologic typing and grading following recommendations of the World Health Organization [11]. In addition, depth of invasion $(\mathrm{T})$, nodal status $(\mathrm{N})$, distant metastasis (M) (was obtained from Oncology files) and staging were assessed according to the American Joint Committee on Cancer [12]

\section{Immunohistochemistry:}

Four micrometer sections of formalin-fixed paraffin-embedded tissue samples of he studied cases were prepared. All sections were incubated with $3 \%$ hydrogen peroxide for $15 \mathrm{~min}$ to block endogenous peroxidase activity at first. The antigen retrieval was performed by high pressure method for $3 \mathrm{~min}$ in citrate buffer (pH 6). Immunohistochemical (IHC) staining was performed using the primary antibody which was rabbit polyclonal antibody, Anti-OTUB 1 (ab 198214) concentrate antibody (Abcam, Cambridge, UK), was applied at the dilution of $1: 100$ for $2 \mathrm{~h}$ at room temperature. After rinsing, staining was performed with Reagent $\mathrm{A}$ and Reagent B subsequently. The color was developed by reacting with Diaminobenzidine (DAB). Sections were then counterstained with hematoxylin, dehydrated, cleared and cover slipped. The positive staining was demonstrated by the presence of obvious brown or yellow particles on a low-power objective. The human breast tissue was used as positive control according to the manufactures instructions.

\section{Interpretation of immunohistochemical staining:}

Positive immunoexpression was defined as cases showing cytoplasmic staining pattern of tumor cells. OTUB ${ }_{1}$ Immunoreactivity Score (IRS) was calculated by the multiply of two values: (A) Staining intensity: No immunoreactivity was scored as 0 , weak immunostaining was 1 , moderate immunostaining was 2 , and strong immunostaining was 3; (B) Percentage of positive tumor cells: 0$5 \%$ was $0,6-25 \%$ was $1,26-75 \%$ was 2 , and 75 $100 \%$ was 3 . The final score of $0-3$ was categorized as low expression, and 4-9 was categorized as high expression [4]

\section{Statistical analysis:}

The collected data was revised, coded, tabulated and introduced to a PC using Statistical package for Social science (SPSS 15.0.1 for windows; SPSS Inc, Chicago, IL, 2001). Data were expressed as mean $( \pm S D)$ for quantitative parametric measures in addition to both number and percentage for categorized data. Chi-square $\left(\chi^{2}\right)$ test was done to study the association between more than one variable as between OTUB ${ }_{1}$ expression and different clinico-pathological parameters of the studied cases. Student $t$-test was used to assess the statistical significance of the difference between two study group means. Fisher's exact test: Was used to examine the relationship between two qualitative variables when the expected count is less than 5 in more than $20 \%$ of cells. $p \leq 0.05$ was considered the cut off value of significant. Differences were considered highly significant when $p \leq 0.001$.

\section{Results}

\section{Clinicopathological results:}

In the present study, the mean age of patients was $50.64 \pm 12.27$ years and female to male ratio was 1.36:1. The pathological features regarding the cases of CRC such as tumor depth, lymph node status, distant metastasis, stage and histologic grading are shown in (Table 1). Most of cases (26 cases; $57.8 \%$ ) were low grade and infiltrating into subserosa or perirectal tissue ( $\left.\mathrm{T}_{3}\right)$, metastasis to 1-3 lymph nodes ( $\left.\mathrm{N}_{1}\right)$ was detected in 20 cases (44.4\%), distant metastasis was present in 19 cases (42.2\%), and stage III and IV were the commonest stages (36 cases; $80 \%$ ).

\section{Immunohistochemical results:}

OTUB $_{1}$ expression was mainly detected in the cell cytoplasm. Non-neoplastic colonic lesions 
showed negative staining and few showed weak focal staining. Immunohistochemical expression of OTUB ${ }_{1}$ is summarized in (Table 2). High OTUB $_{1}$ expression was found in $57.8 \%$ of the studies cases while low OTUB 1 expression was found in $42.2 \%$ of cases (Table 2), Graph (1).

High OTUB 1 expression was statistically associated with high grade tumors $(p<0.001)$, depth T $(p=0.03)$ and location of CRC $(p=0.002)$.

$69.2 \%$ of cases with high OTUB 1 expression were associated with high grade tumors Graph (2). Mean OTUB ${ }_{1}$ expression in high grade tumors

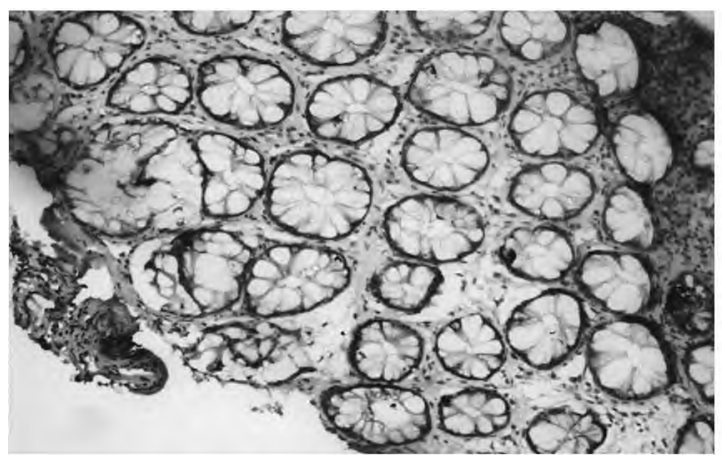

Fig. (1): OTUB 1 staining of non-neoplastic colonic mucosa shows negative staining (IHC X200).

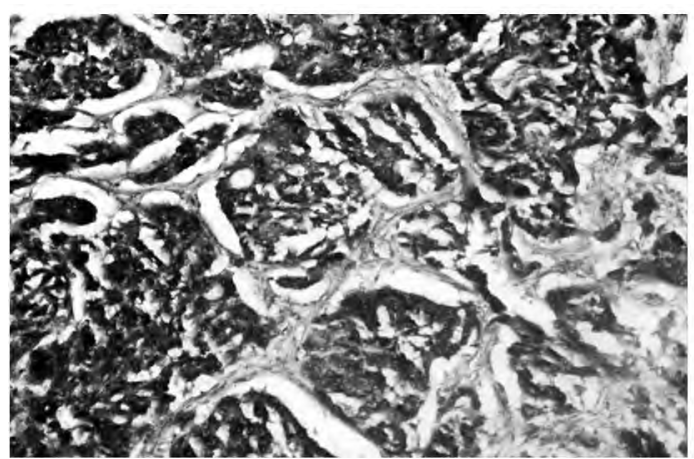

Fig. (3): A case of high grade colonic adenocarcinoma shows high expression of OTUB 1 (IHC X200).

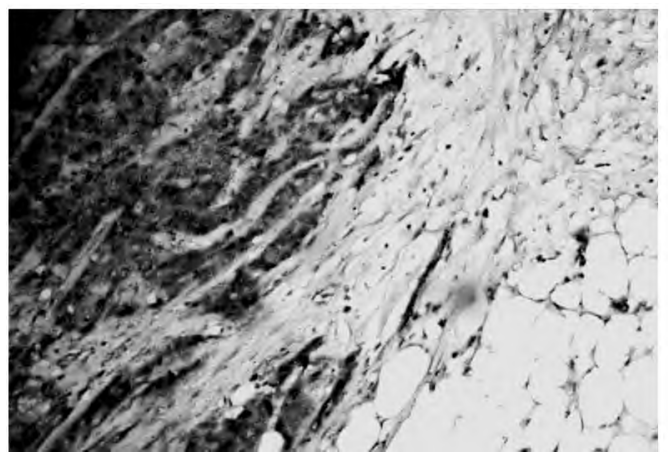

Fig. (5): A case of high grade colonic adenocarcinoma infiltrating up to serosal fat $\left(\mathrm{T}_{3}\right)$ shows high expression of OTUB 1 (IHC X200).
(7.21) was significantly higher than that of low grade tumors (2.77) Graph (3). Most of high grade tumors expressed the marker with score > 6 with sensitivity (84.2\%) and specificity (73.1\%) Graph (4). $88.5 \%$ of cases with high OTUB1 expression were associated with greater tumor depth $\mathrm{T}_{3}$ and $\mathrm{T}_{4}$ Graph (5).

No statistically significant relationship was detected as regards age ( $p=0.93)$, gender $(p=0.99)$, lymph node metastasis $(p=0.36)$, distant metastasis $(p=0.22)$, TNM stage $(p=0.12)$, size of tumor $(p=$ 0.5) (Table 3).

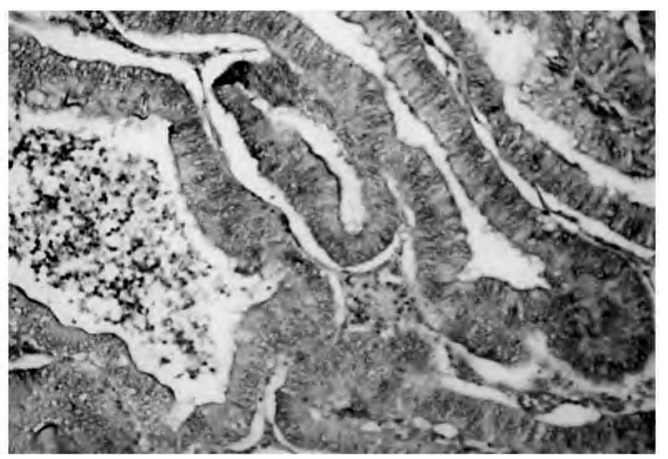

Fig. (2): A case of low grade colonic adenocarcinoma shows low expression of OTUB 1 (IHC X200).

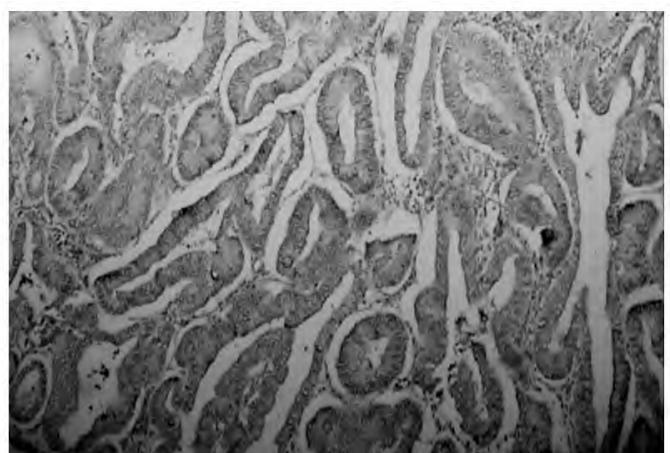

Fig. (4): A case of low grade colonic adenocarcinoma invades submucosa $\left(\mathrm{T}_{1}\right)$ shows low expression of OTUB (IHC X100).

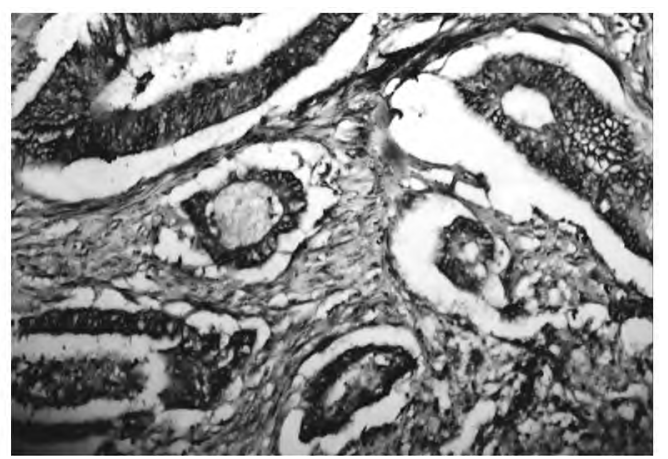

Fig. (6): A case of low grade colonic adenocarcinoma invades urinary bladder $\left(\mathrm{T}_{4}\right)$ shows high expression of OTUB (IHC X200). 
Table (1): Clinicopathological parameters.

\begin{tabular}{|c|c|c|c|c|}
\hline \multicolumn{2}{|c|}{ N Minimum } & Maximum & Mean & SD \\
\hline \multirow[t]{2}{*}{ Age 45} & 23.00 & 70.00 & 50.64 & 12.27 \\
\hline & \multicolumn{2}{|c|}{ Count } & \multicolumn{2}{|c|}{ Percentage } \\
\hline \multicolumn{5}{|l|}{ Gender: } \\
\hline Males & \multicolumn{2}{|c|}{19} & \multicolumn{2}{|c|}{42.2} \\
\hline Females & \multicolumn{2}{|c|}{26} & \multicolumn{2}{|c|}{57.8} \\
\hline Total & \multicolumn{2}{|c|}{45} & \multicolumn{2}{|c|}{100.0} \\
\hline
\end{tabular}

Count Minimum Maximum Mean SD

\begin{tabular}{llllll}
\hline Tumor sixe $(\mathrm{cm})$ & 45 & 2.00 & 13.00 & 4.69 & 2.43 \\
\hline
\end{tabular}

\begin{tabular}{lcc}
\hline Site & Count & Percentage \\
\hline Right sided & 19 & 42.22 \\
Left sided & 26 & 57.78 \\
Total number & 45 & 100 \\
\hline & Count & $\%$ \\
\hline
\end{tabular}

Grading:

$\begin{array}{lrr}\text { Low } & 26 & 57.8 \\ \text { High } & 19 & 42.2\end{array}$

Depth:

$\begin{array}{lll}\mathrm{T}_{1} & 2 & 4.4 \\ \mathrm{~T}_{2} & 8 & 17.8 \\ \mathrm{~T}_{3} & 26 & 57.8 \\ \mathrm{~T} 4 & 9 & 20.0\end{array}$

$L N$ :

$\begin{array}{lll}\mathrm{N}_{0} & 14 & 31.2 \\ \mathrm{~N}_{1} & 20 & 44.4 \\ \mathrm{~N} 2 & 11 & 24.4\end{array}$

Metastasis:

$\begin{array}{lll}\mathrm{M}_{0} & 26 & 57.8\end{array}$

$\begin{array}{lll}\text { M1 } & 19 & 42.2\end{array}$

Stage:

$\begin{array}{lll}\text { I } & 5 & 11.1\end{array}$

$\begin{array}{lll}\text { II } & 4 & 8.9\end{array}$

III $\quad 18 \quad 40.0$

$\begin{array}{lll}\text { IV } & 18 & 40.0\end{array}$

Total number

45

100

Table (2): Distribution of OTUB 1 expression in the studied cases.

\begin{tabular}{|c|c|c|c|c|c|}
\hline & $\mathrm{N}$ & imum & Maximum & Mean & SD \\
\hline \multirow[t]{2}{*}{ Score } & 45 & 0.00 & 9.00 & 4.64 & 3.45 \\
\hline & & \multicolumn{2}{|r|}{ Count } & \multicolumn{2}{|c|}{ Percentage } \\
\hline \multicolumn{6}{|c|}{ OTUB $_{1}$ grade: } \\
\hline \multicolumn{2}{|l|}{ Low } & \multicolumn{2}{|r|}{19} & \multicolumn{2}{|c|}{42.2} \\
\hline \multicolumn{2}{|l|}{ High } & \multicolumn{2}{|r|}{26} & \multicolumn{2}{|c|}{57.8} \\
\hline \multicolumn{2}{|c|}{ Total } & \multicolumn{2}{|r|}{45} & \multicolumn{2}{|c|}{100.0} \\
\hline
\end{tabular}

Table (3): Relation between OTUB 1 expression and clinicopathologic data.

\begin{tabular}{|c|c|c|c|c|c|c|}
\hline & & & & & & \\
\hline & & & & & $t^{*}$ & $p-$ \\
\hline & Mean & SD & Mean & SD & & \\
\hline Age & 50.84 & 13.12 & 50.50 & 11.87 & 0.09 & 0.93 \\
\hline & & & DTUB1 & & & \\
\hline & & Low & & High & & $p-$ \\
\hline & Cou & Perce & ge $\mathrm{Cou}$ & Perce & & \\
\hline
\end{tabular}

\begin{tabular}{|c|c|c|c|c|c|c|}
\hline \multicolumn{7}{|l|}{ Gender: } \\
\hline Male & 8 & 42.1 & 11 & 42.3 & \multirow[t]{2}{*}{0.00} & 0.99 \\
\hline Female & 11 & 57.9 & 15 & 57.7 & & \\
\hline \multicolumn{5}{|c|}{ OTUB1 } & \multirow{3}{*}{$x^{2} *$} & \multirow{3}{*}{$\begin{array}{c}p- \\
\text { value }\end{array}$} \\
\hline \multicolumn{3}{|c|}{ Low } & \multicolumn{2}{|r|}{ High } & & \\
\hline $\mathrm{Cou}$ & & entage & Count & Percentage & & \\
\hline
\end{tabular}

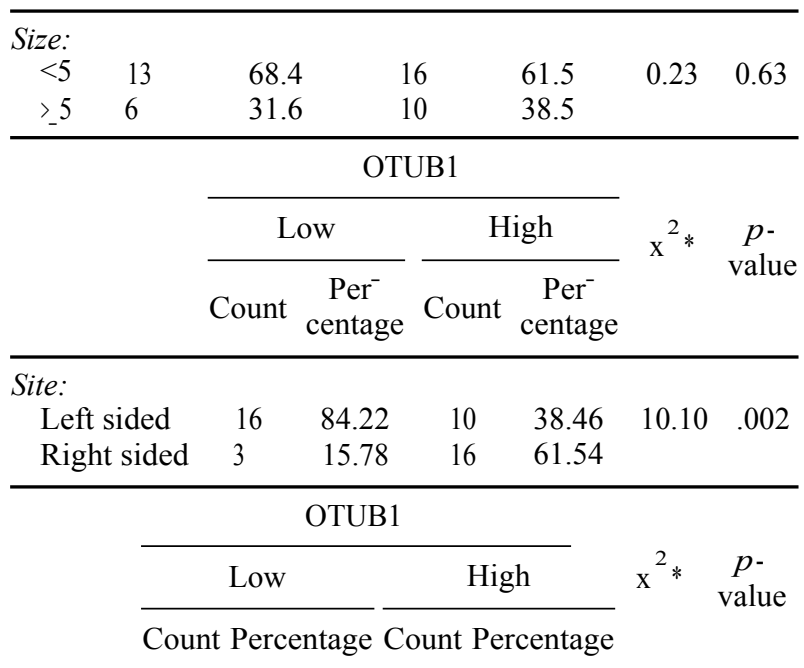

\begin{tabular}{|c|c|c|c|c|c|c|}
\hline $\begin{array}{c}\text { Grading: } \\
\text { Low } \\
\text { High }\end{array}$ & $\begin{array}{l}18 \\
1\end{array}$ & $\begin{array}{l}94.7 \\
5.3\end{array}$ & $\begin{array}{l}8 \\
18\end{array}$ & $\begin{array}{l}30.8 \\
69.2\end{array}$ & 18.41 & $<0.001$ \\
\hline & \multicolumn{3}{|c|}{ OTUB1 } & \multirow{2}{*}{$t^{*}$} & \multirow{2}{*}{\multicolumn{2}{|c|}{$p$-value }} \\
\hline & \multicolumn{2}{|c|}{ Mean } & SD & & & \\
\hline \multicolumn{7}{|l|}{ Grading: } \\
\hline Low & \multicolumn{2}{|c|}{2.77} & 2.76 & \multirow{2}{*}{\multicolumn{2}{|c|}{5.52}} & \multirow[t]{2}{*}{$<0.001$} \\
\hline \multirow[t]{4}{*}{ High } & & & 2.53 & & & \\
\hline & \multicolumn{4}{|c|}{ OTUB1 } & \multirow{3}{*}{$x^{2} *$} & \multirow{3}{*}{$\begin{array}{c}p- \\
\text { value }\end{array}$} \\
\hline & \multicolumn{2}{|c|}{ Low } & \multicolumn{2}{|c|}{ High } & & \\
\hline & Count & $\%$ & Count & $\%$ & & \\
\hline \multicolumn{7}{|l|}{ Depth: } \\
\hline $\mathrm{T}_{1}$ & 2 & 10.5 & 0 & 0.0 & \multirow{4}{*}{$\begin{array}{l}5.15 \\
\text { Fisher } \\
\text { exact }\end{array}$} & \multirow[t]{4}{*}{0.03} \\
\hline $\mathrm{T}_{2}$ & 5 & 26.3 & 3 & 11.5 & & \\
\hline $\mathrm{T}_{3}$ & 10 & 52.6 & 16 & 61.5 & & \\
\hline T4 & 2 & 10.5 & 7 & 26.9 & & \\
\hline \multicolumn{7}{|l|}{ Depth: } \\
\hline $\mathrm{T} 1 / \mathrm{T} 2$ & 7 & 36.8 & 3 & 11.5 & \multirow[t]{2}{*}{4.07} & \multirow[t]{2}{*}{0.04} \\
\hline T3/T4 & 12 & 63.2 & 23 & 88.5 & & \\
\hline
\end{tabular}


Table (3): Count.

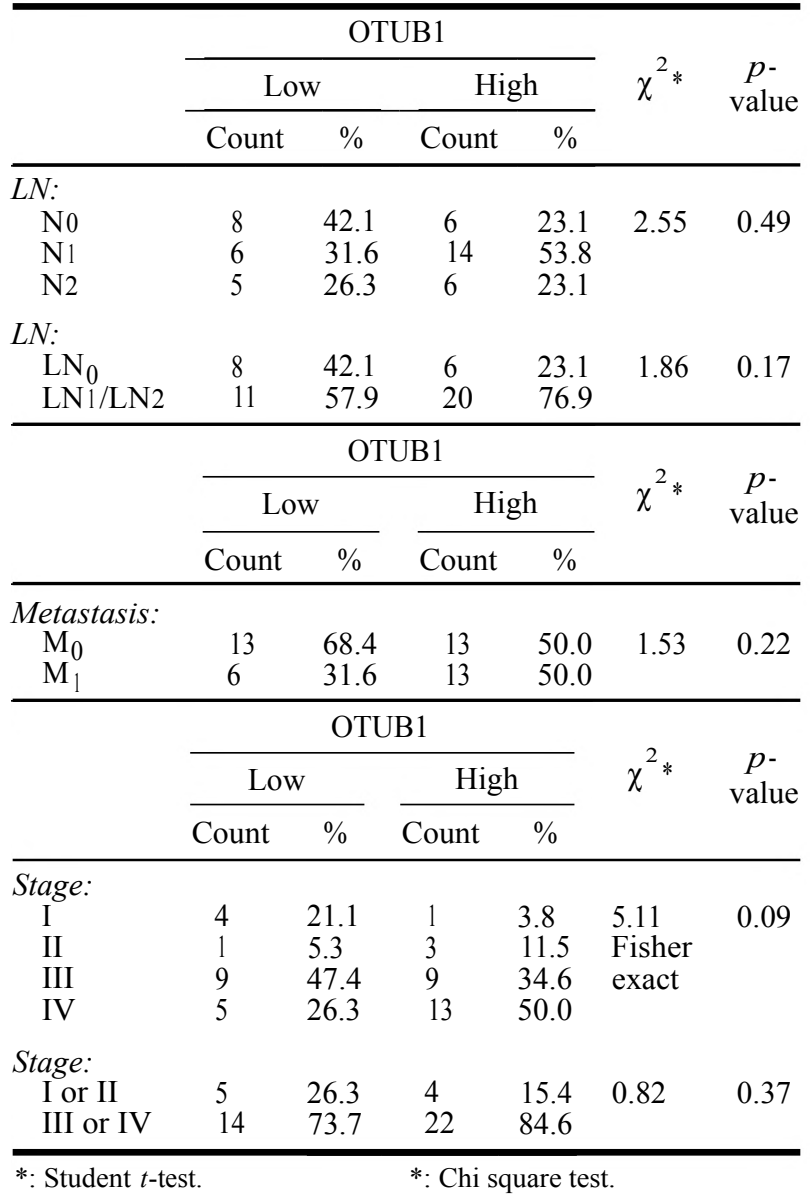

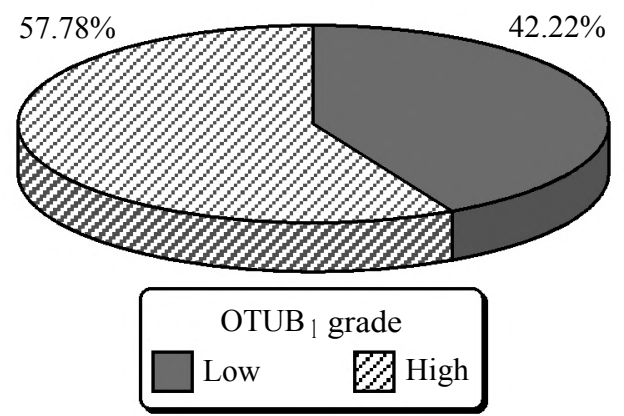

Graph (1): Distribution of OTUB ${ }_{1}$ expression in the studied cases.

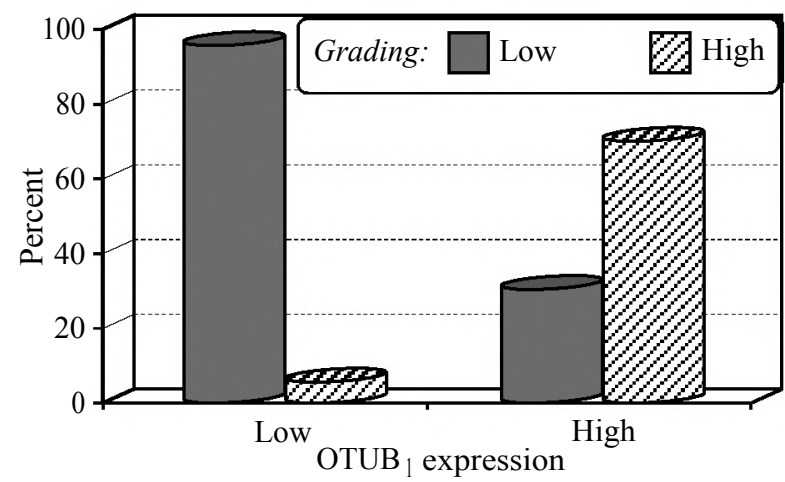

Graph (2): Immunohistochemical expression of OTUB ${ }_{1}$ and histopathological grading of tumor.

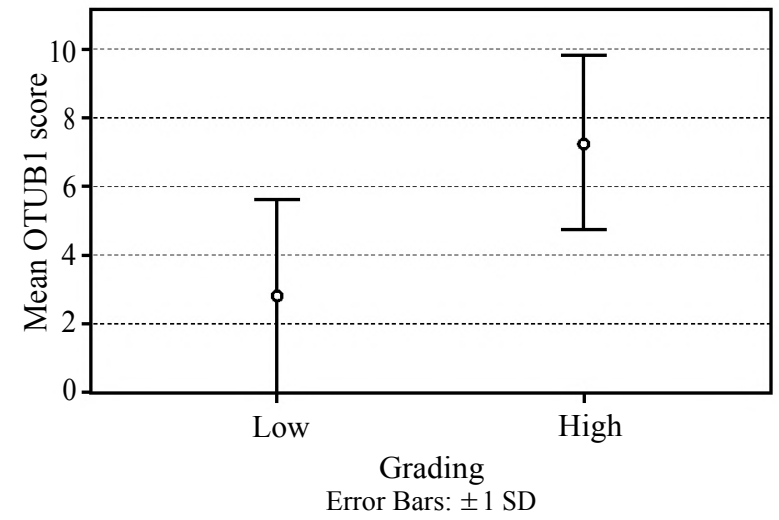

Graph (3): Mean of OTUB1 score and histopathological grading \pm 1 SD. Mean OTUB1 expression in low grade tumors was $2.77 \pm 1$ SD while was $7.21 \pm 1$ $\mathrm{SD}$ in high grade tumors.

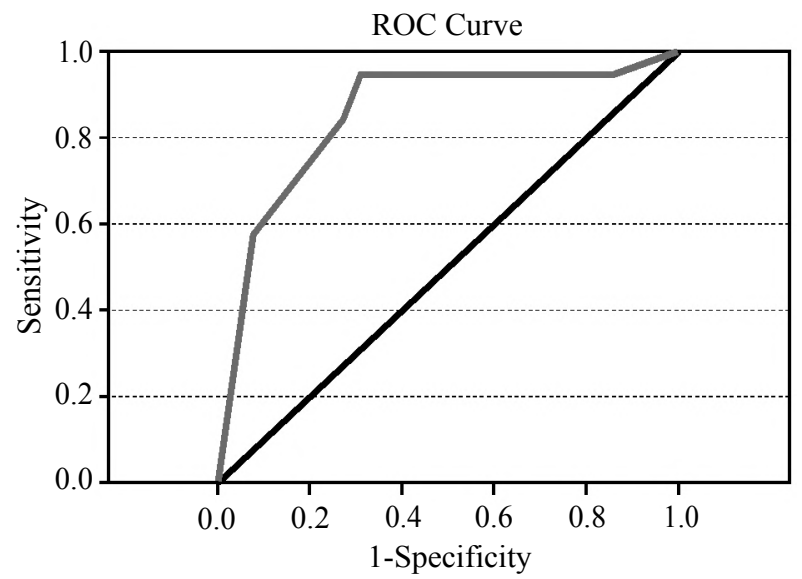

Diagonal segments are produced by ties

Area under the curve

Test result variable (s): OTUB ${ }_{1}$ score

\begin{tabular}{ccccc}
\hline & & \multicolumn{2}{c}{$95 \%$ confidence interval } \\
\cline { 3 - 5 } Area & Std. error & Sig. & Lower Bound & Upper Bound \\
\hline 0.853 & 0.062 & $<0.001$ & 0.733 & 0.974 \\
\hline
\end{tabular}

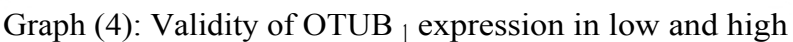
grades of tumor.

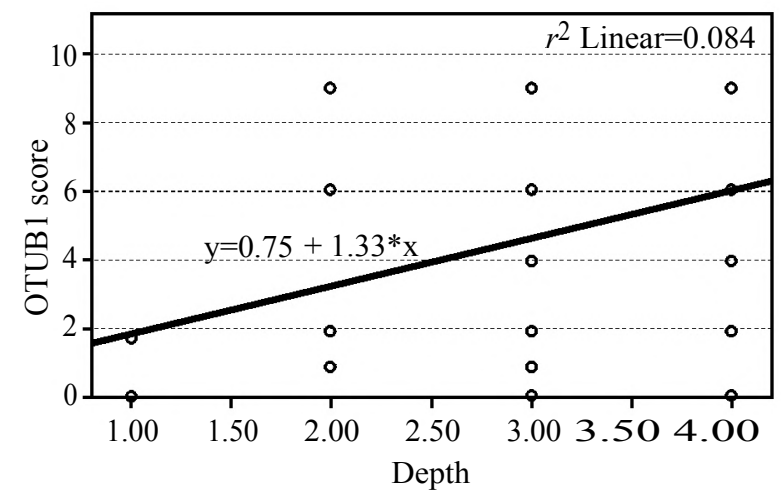

Graph (5): Correlation between OTUB 1 expression and depth of tumor. 


\section{Discussion}

OTUB $_{1}$ gene is located in human chromosome

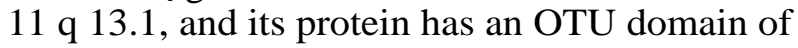
130 amino acids, which are highly conserved from yeast to mammals [13]. OTUB 1 has recently emerged as a unique atypical DUB that inhibits DNA repair without reflecting DUB activity per se [5].

A limited number of studies have provided conflicting results regarding the functions of

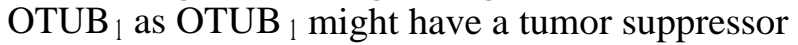
function by inducing p53 dependent apoptosis and inhibited cell growth [14]. However OTUB ${ }_{1}$ also played crucial roles in transforming growth factor$\beta$ (TGF- $\beta$ mediated) gene transcription and cellular migration by stabilizing SMAD 2/3 which drives cell invasion in somatic cells, suggesting that it might be involved in tumor migration [7]

In this study, higher expression level in CRC tissues was obviously noted compared to the nonneoplastic colonic mucosa. Thereby, OTUB 1 seems to play a role in tumor initiation: This is supported by [9] who reported a positive correlation between OTUB $_{1}$ and nuclear $(3$-catenin expression which increases from early adenoma to carcinomas [15]

In this work, high OTUB 1 staining was detected in twenty six of CRC cases representing (57.8\%), and low OTUB ${ }_{1}$ expression was observed in nineteen of CRC patients constituting (42.2\%). This is compatible with what was reported by Liu et al., who observed high OTUB 1 expression in (62\%) of cases and low OTUB ${ }_{1}$ expression in (38\%) [4], and $\mathrm{Ni}$ et al., also reported that high OTUB $\mathrm{ex}$ pression in $(61 \%)$ of cases and low OTUB ${ }_{1}$ expression in $(39 \%)$ [16].

There was no significant relationship between the OTUB ${ }_{1}$ expression and demographic data such as age and gender in our study. These results are compatible with Liu et al., Zhou et al., and Ni et al., $[4,9,16]$.

No statistically significant correlation between OTUB $_{1}$ expression and size of CRC in our study. Our results were in accordance with that of Zhou et al., and Ni et al., in CRC and Hepatocellular Carcinoma (HCC), respectively $[\mathbf{9 , 1 6}]$

In our study there was statistically significant correlation between right and left colon as higher OTUB 1 expression was observed in right sided of colon as compared to left sided. Zhou et al., [9] reported that there was no statistically significant correlation between the site of $\mathrm{CRC}$ whether colonic or rectal carcinoma and the expression of OTUB ${ }_{1}$

The present study showed that OTUB 1 expression significantly correlated with tumor differentiation, indicating that OTUB ${ }_{1}$ is an advert factor of prognosis. The frequency of OTUB overexpression was $30.8 \%$ and $69.2 \%$, respectively, in low grade and high grade cancer tissues. These results are in accordance with Liu et al., and Ni et al., who reported positive correlation of OTUB ${ }_{1}$ expression and degree of differentiation in CRC and HCC, respectively $[4,16]$

On the contrary, Weng et al., reported that there was no significant correlation between OTUB expression and degree of differentiation in invasive gastric carcinoma [17] .

Our results revealed that the expression level of OTUB 1 was significantly associated with tumor invasive, as OTUB ${ }_{1}$ overexpression in $\mathrm{T}_{1} / \mathrm{T}_{2}$ was $11.5 \%$ while in $\mathrm{T}_{3} / \mathrm{T}_{4}$ was $88.5 \%$. Similar results were obtained by Zhou et al., Weng et al., and Baietti et al., who reported positive correlation between the OTUB ${ }_{1}$ expression and depth in CRC, invasive gastric carcinoma and extension in lung carcinoma, respectively $[\mathbf{9 , 1 0 , 1 7 ]}$. Consistent with these results, Liu et al., reported that the expression of USP22 (another DUBs member) was highly correlated with the depth in CRC [4] .

In this study, OTUB ${ }_{1}$ expression was not correlated with lymph node metastasis. On the contrary studies obtained by Liu et al., and Zhou et al., reported significant difference $[4,9]$. It may be attributed to the difference in the sample size between our study and these two previous studies.

Regarding distant metastasis, Zhou et al., and $\mathrm{Ni}$ et al., reported a positive correlation between OTUB $_{1}$ expression and distant metastasis in CRC and HCC, respectively $[\mathbf{9 , 1 6}$. Zhou et al., demonstrated that OTUB 1 could promote metastasis through inhibition or loss of E-cadherin in CRC cell lines [9].

On the other hand, no statistically significant association between OTUB 1 expression and distant metastasis in our work. This is compatible with Liu et al., Wang et al., and Weng et al., who also reported no significant correlation between OTUB 1 expression and distant metastasis in CRC, ovarian and gastric carcinoma, respectively $[4,17,18]$. Thus, we propose that although OTUB 1 might be involved in local tumor progression and dedifferentiation. It seems that it might not have a direct role in metastasis whether nodal or distant. 
In this study no statistically significant association between OTUB1 expression and the TNM staging, this is compatible with Zhou et al., who found that OTUB 1 expression was not associated with stage II, III CRC patients [9]. Karunarathna et al., also reported no significant association between OTUB ${ }_{1}$ expression and breast cancer staging [19]

On the other hand, Weng et al., and Ni et al., reported significant association between OTUB expression and staging in gastric carcinoma and HCC, respectively [16,17].

Such controversy highlights the need of further studies on larger scale to elucidate the relation OTUB $_{1}$ and each of nodal metastasis, distant metastasis and tumor stage. This, in turn, may help to determine whether or not OTUB 1 could serve as a biomarker of metastasis and consequently advanced stage.

In conclusion, the present study demonstrated that OTUB ${ }_{1}$ is overexpressed in CRC, and its expression level is related with some clinicopathologic parameters. The result indicated OTUB may be responsible for the tumor development especially in the right sided CRC and invasion. High OTUB ${ }_{1}$ expression in CRC cases especially with a score $\geq 6$ could be useful in identification of biologically aggressive tumors in colonoscopic biopsies. OTUB 1 could be an attractive target for the development of new strategies in the diagnosis and treatment of CRC in order to reduce local invasiveness pre-operatively.

\section{References}

1- ARNOLD M., SIERRA M.S., LAVERSANNE M., et al. Global patterns and trends in colorectal cancer incidence and mortality. Gut., 66 (4): 683-91, 2017.

2- ZEENELDIN A.A., SABER M.M., SEIF EL-DIN I.A. and FRAG S.A.: Colorectal carcinoma in gharbiah district, Egypt: Comparison between the elderly and non-elderly. J. Solid. Tumor, 2 (3): 13-23, 2012.

3- LAO V.V. and GRADY W.M.: Epigenetics and colorectal cancer. Nat. Rev. Gastroenterol. Hepatol., 8: 686-700, 2011.

4- LIU X., JIANG W.N., WANG J.G. and CHEN H.: Colon cancer bears overexpression of OTUB 1 . Pathol. Res. Pract., 210: 770-3, 2014.

5- WIENER R., DiBELLO A.T., LOMBARDI P.M., et al.: E2 ubiquitin-conjugating enzymes regulate the deubiquitinating activity of OTUB 1 . Nat. Struct. Mol. Biol., 20 (9): 1033-9, 2013.

6- GONCHARV T., NIESSEN K., De ALMAGRO M.C., et al.: OTUB 1 modulates c-IAP1 stability to regulate signaling pathways. EMBO Journal, 32 (8): 1103-14, 2013.
7- HERHAUS L., AL-SALIHI M., MACARTNEY T., et al.: OTUB 1 enhances TGF $\beta$ signalling by inhibiting the ubiquitylation and degradation of active SMAD2/3. Nat. Commun., 4: 2519, 2013.

8- LI Y., SUN X.X., ELFERICH J., et al.: Monoubiquitination is critical for ovarian tumor domain-containing ubiquitin aldehyde binding protein 1 (OTUB 1 ) to suppress UbcH5 enzyme and stabilize p53 protein. J. Biol. Chem., 289 (8): 5097-108, 2014.

9- ZHOU Y., WU J., FU X., DU W., ZHOU L., MENG X., et al.: OTUB 1 promotes metastasis and serves as a marker of poor prognosis in colorectal cancer. Mol. Cancer, 13: $258,2014$.

10- BAIETTI M.F., SIMICEK M., ABBASI ASBAGH L., RADAELLI E., LIEVENS S., CROWTHER J., STEKLOV M., AUSHEV V.N., MARTINEZ GARCIA D., TAVERNIER J. and SABLINA A.A.: OTUB 1 triggers lung cancer development by inhibiting RAS monoubiquitination. E.M.B.O. Mol. Med., 8: 288-303, 2016.

11- HAMILTON S.R., BOSMAN F.T., BOFFETTA P., et al.: Carcinoma of the colon and rectum. In: Bosman F.T., Carneiro F., Hruban R.H., Theise N.D., editors. WHO Classification of Tumours of the Digestive System. $4^{\text {th }}$ ed. Lyon: IARC, 134-46, 2010.

12- EDGE S.B. and COMPTON C.C.: The American Joint Committee on Cancer: The 7 th Edition of the AJCC Cancer Staging Manual and the Future of TNM. Ann. Surg. Oncol., 17 (6): 1471-4, 2010.

13- FRIAS-STAHELI N., GIANNAKOPOULOS N.V., KIKKERT M., TAYLOR S.L., BRIDGEN A., PARAGAS J., et al.: Ovarian tumor domain-containing viral proteases evade ubiquitin-and ISG15-dependent innate immune responses. Cell Host. Microbe, 2 (6): 404-16, 2007.

14- SUN X.X., CHALLAGUNDLA K.B. and DAI M.S.: Positive regulation of p53 stability and activity by the deubiquitinating enzyme Otubain 1. E.M.B.O. J., 31: 57692, 2012.

15- BRABLETZ T., HERRMANN K., JUNG A., FALLER G. and KIRCHNER T.: Expression of nuclear beta-catenin and c-myc is correlated with tumor size but not with proliferative activity of colorectal adenomas. Am. J. Pathol., 156: 865-70, 2000.

16- NI Q., CHEN J., LI X., XU X., ZHANG N., ZHOU A., ZHOU B., LU Q. and CHEN Z.: Expression of OTUB in hepatocellular carcinoma and its effects on HCC cell migration and invasion. Acta. Biochim. Biophys. Sin. (Shanghai), 1-9, 2017.

17- WENG W., ZHANG Q., XU M., et al.: OTUB 1 promotes tumor invasion and predicts a poor prognosis in gastric adenocarcinoma. American Journal of Translational Research, 8 (5): 2234-44, 2016.

18- WANG Y., ZHOU X., XU M., WENG W., ZHANG Q., YANG Y., WEI P. and DU X.: OTUB 1 -catalyzed deubiquitination of FOXM1 facilitates tumor progression and predicts a poor prognosis in ovarian cancer. Oncotarget, 7: 36681-97, 2016

19- KARUNARATHNA U., KONGSEMA M., ZONA S., GONG C., CABRERA E., GOMES A.R., MAN E.P., KHONGKOW P., TSANG J.W., KHOO U.S., MEDEMA R.H., FREIRE R. and LAM E.W.: OTUB 1 inhibits the ubiquitination and degradation of FOXM1 in breast cancer and epirubicin resistance. Oncogene, 35: 1433-44, 2016. 


\section{الظهور الهيستولوجى الكيميائى المناعى للإنزيم المسئول عن إزاله

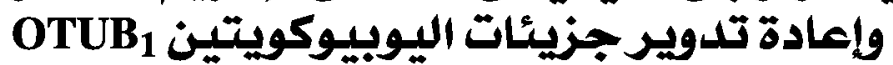 فى حالات سرطان القولون والمستقيئ اليوينيم}

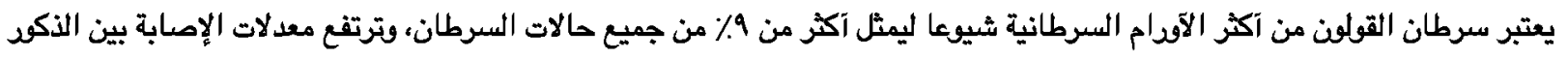

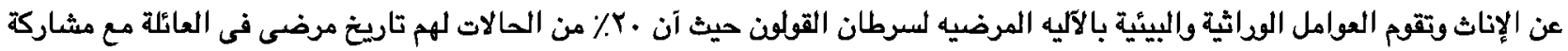

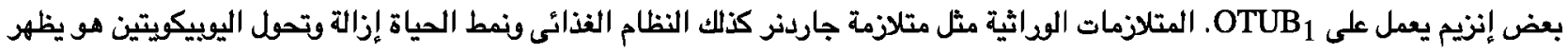

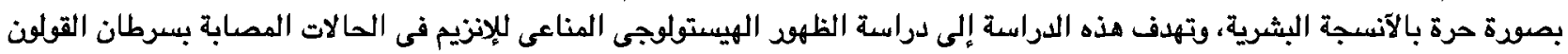

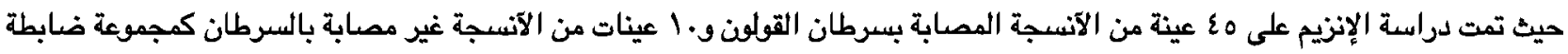

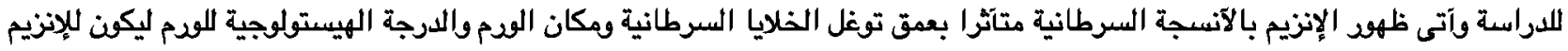
لود محتمل فى التعرف على عدائية النسيج السرطانى وإختيار نظام العلاج اللحالات المصابة. 